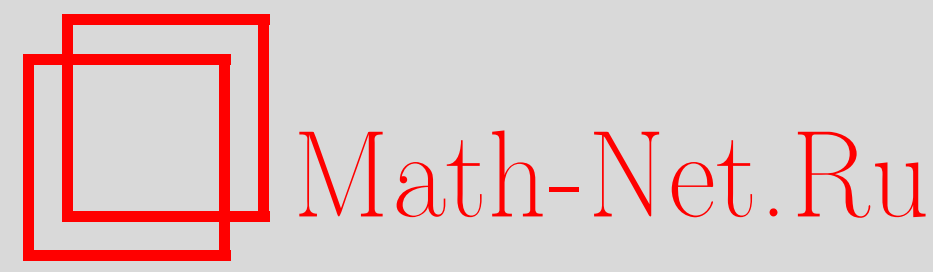

Ю. Н. Субботин, С. А. Теляковский, Асимптотика констант Лебега периодических интерполяционных сплайнов с равноотстоящими узлами, Матем. сб., 2000, том 191, номер 8, 131-140

DOI: https://doi.org/10.4213/sm502

Использование Общероссийского математического портала Math-Net.Ru подразумевает, что вы прочитали и согласны с пользовательским соглашением

http://www. mathnet.ru/rus/agreement

Параметры загрузки:

IP : 3.93 .64 .190

26 апреля 2023 г., 09:06:04 


\section{Асимптотика констант Лебега периодических интерполяционных сплайнов с равноотстоящими узлами}

\footnotetext{
Каждой непрерывной функции $f$ периода 1 ставится в соответствие периодический сплайн $s_{r, n}(f)$ степени $r$ дефекта 1 с узлами в точках $x_{i}=i / n$, $i=0,1, \ldots, n-1$, интерполирующий $f$ в узлах $x_{i}$, если $r$ нечетно, и в серединах отрезков $\left[x_{i}, x_{i+1}\right]$, если $r$ четно.

Для констант Лебега $L_{r, n}$ этого процесса, т.е. для норм оператора $f(x) \rightarrow$ $s_{r, n}(f)$ как оператора из $C$ в $C$, установлена асимптотическая формула

$$
L_{r, n}=\frac{2}{\pi} \log \min (r, n)+O(1)
$$
}

равномерная относительно значений $r$ и $n$.

Библиографофия: 6 названий.

\section{§1. Введение}

На протяжении всей работы $r$ и $n$ будут обозначать натуральные числа, $r \geqslant 1$, $n \geqslant 3$, a $\nu:=\left[\frac{n-1}{2}\right]$. Мы не будем в дальнейшем напоминать об этих соглашениях.

Заметим, что из определения числа $\nu$ следует, что

$$
1 \leqslant n-2 \nu \leqslant 2
$$

Будем рассматривать на отрезке $[0,1]$ периодические с периодом 1 сплайны степени $r$ дефекта 1 с узлами в $n$ равноотстоящих точках $x_{i}=i / n, i=0,1, \ldots, n-1$.

Для каждой 1-периодической непрерьвной функции $f$ построим сплайн указанного вида $s_{r, n}(f, x)$, интерполирующий $f$ в точках

$$
t_{i}:=x_{i}+\frac{1+(-1)^{r}}{4 n}
$$

Существование и единственность интерполяционных сплайнов $s_{r, n}(f, x)$ известны (см. [1]).

Константы Лебега интерполяционных сплайнов, т.е. нормы $s_{r, n}(f, x)$ как операторов из $C$ в $C$, будем обозначать $L_{r, n}$.

Настоящая работа посвящена выяснению асимптотического поведения констант Лебега $L_{r, n}$ как функции растущих параметров $r$ и $n$.

Работа вьполнена при финансовой поддержке Российского фонда фундаментальных исследований - Государственного фонда естественных наук Китая (грант № 99-01-39006). 
ТЕОРема. Для констант Лебега периодических интерполяционных сплайнов с равноотстоящими узлами справедлива оценка

$$
L_{r, n}=\frac{2}{\pi} \log \min (r, n)+O(1)
$$

с абсолютной постоянной в остаточном члене.

При доказательстве теоремы мы будем пользоваться следующим представлением констант Лебега $L_{r, n}$, установленным А. А. Женськбаевым [2; теорема 2]:

$$
L_{r, n}=\frac{2+(-1)^{\nu}+(-1)^{\nu+n}}{2 n}+\frac{1}{n} \sum_{j=1}^{\nu} \frac{2+(-1)^{j+\nu}+(-1)^{j+\nu+n}}{\cos \frac{j \pi}{n}} \frac{B_{r, j, n}}{A_{r, j, n}},
$$

где

$$
A_{r, j, n}=\sum_{m=-\infty}^{\infty} \frac{(-1)^{m(r+1)}}{(m n+j)^{r+1}}, \quad B_{r, j, n}=\sum_{m=-\infty}^{\infty} \frac{(-1)^{m r}}{(m n+j)^{r+1}} .
$$

Наши обозначения величин $A_{r, j, n}$ и $B_{r, j, n}$ отличаются от принятых в [2] на множитель $(-1)^{r+1}$.

$\Phi$. Ричардс [3] получил другое представление для величин $L_{r, n}$ и показал, что существует предел

$$
\lim _{n \rightarrow \infty} L_{r, n}=L_{r}
$$

При этом, если рассматривать непериодические сплайны степени $r$ дефекта 1 , заданные на всей оси, с узлами в целых точках, интерполирующие непрерывные ограниченные функции в целых точках, если $r$ нечетно, и в точках $i+\frac{1}{2}$, $i=0, \pm 1, \pm 2, \ldots$, если $r$ четно, то величины $L_{r}$ представляют собой константы Лебега этого интерполяционного оператора как оператора из $C$ в $C$.

Уточнив свою оценку $L_{r}=O(\log (r+1))$ из работы [2; следствие], Женсыкбаев [4] показал, что при $r \rightarrow \infty$

$$
L_{r}=\frac{2}{\pi} \log r+O(1) .
$$

Пользуясь сушествованием предела (1.4), этот результат можно получить из оценки (1.2).

Сопоставление оценок (1.5) и (1.2) показывает, как изменяются константы Лебега, если рассматривать нормы интерполяционных сплайнов с узлами в целых точках не на всем классе непрерывных ограниченных на оси функций, а только для функций периода $n$.

Отметим, что, опираясь на результаты из работы [3], Ричардс [5; теорема 1] установил, что при $r \rightarrow \infty$

$$
L_{r}=\frac{2}{\pi} \log r+\frac{2}{\pi}\left(2 \log \frac{4}{\pi}+\gamma\right)+o(1)
$$

где $\gamma$ - постоянная Эйлера. Подобный результат получил также Г. Майнардус [6; теорема 2]; правда, он рассматривал только нечетные $r$. Но вместе с тем, в [6] остаточный член в формуле (1.6) записан в виде $O\left(r^{-1}\right)$.

В настояшей работе все оценки с $O$-символами являются равномерными относительно всех параметров, но отмечать это будем только в формулировках утверждений. 


\section{§ 2. Вспомогательные предложения}

ЛЕМма 1. Для $j=1, \ldots, n-1$ справедливъ оценки

$$
\frac{1}{j^{r+1}}<A_{r, j, n}<\frac{1}{j^{r+1}}+\frac{4}{(n-j)^{r+1}} .
$$

ДокаЗАТЕЛЬСтво. Имеем

$$
\begin{aligned}
A_{r, j, n} & =\frac{1}{j^{r+1}}+\sum_{m=1}^{\infty} \frac{(-1)^{m(r+1)}}{(m n+j)^{r+1}}+\sum_{m=1}^{\infty} \frac{(-1)^{-m(r+1)}}{(-m n+j)^{r+1}} \\
& =\frac{1}{j^{r+1}}+\sum_{m=1}^{\infty}(-1)^{(m+1)(r+1)}\left[\frac{1}{(m n-j)^{r+1}}+(-1)^{r+1} \frac{1}{(m n+j)^{r+1}}\right] .
\end{aligned}
$$

Так как значения выражений в квадратных скобках с ростом $m$ убывают, то сумма по $m$ в правой части (2.2) положительна, что доказывает левую оценку (2.1).

Далее, в силу (2.2) имеем

$$
\begin{aligned}
A_{r, j, n} & <\frac{1}{j^{r+1}}+\sum_{m=1}^{\infty} \frac{2}{(m n-j)^{r+1}}<\frac{1}{j^{r+1}}+\sum_{m=1}^{\infty} \frac{2}{(m n-m j)^{r+1}} \\
& \leqslant \frac{1}{j^{r+1}}+\frac{2}{(n-j)^{r+1}} \sum_{m=1}^{\infty} \frac{1}{m^{2}}
\end{aligned}
$$

откуда вытекает правая оценка (2.1).

Лемма доказана.

ЛЕмма 2. Для величин $B_{r, j, n}$ при $j=1, \ldots, \nu$ справедливы оценки

$$
0<B_{r, j, n}=\frac{1}{j^{r+1}}-\frac{1}{(n-j)^{r+1}}+O\left(\frac{n-2 j}{n j^{r+1}}\right)
$$

с абсолютной постоянной в остаточном члене.

ДокАЗАТЕЛЬСТво. Имеем

$$
\begin{aligned}
B_{r, j, n}= & \sum_{m=-\infty}^{\infty}(-1)^{m r}\left(\frac{2 m+1}{2} n-\frac{n-2 j}{2}\right)^{-(r+1)} \\
= & \sum_{m=0}^{\infty}(-1)^{m r}\left(\frac{2 m+1}{2} n-\frac{n-2 j}{2}\right)^{-(r+1)} \\
& +\sum_{m=0}^{\infty}(-1)^{-(m+1) r}\left(\frac{-2(m+1)+1}{2} n-\frac{n-2 j}{2}\right)^{-(r+1)} \\
= & \sum_{m=0}^{\infty}(-1)^{m r}\left[\left(\frac{2 m+1}{2} n-\frac{n-2 j}{2}\right)^{-(r+1)}-\left(\frac{2 m+1}{2} n+\frac{n-2 j}{2}\right)^{-(r+1)}\right] .
\end{aligned}
$$


Отсюда следует положительность величин $B_{r, j, n}$. Далее, слагаемое, соответствуюшее в этой сумме $m=0$, дает

$$
\frac{1}{j^{r+1}}-\frac{1}{(n-j)^{r+1}} .
$$

Поэтому остается показать, что сумма ряда по $m$ от 1 до $\infty$ оценивается остаточным членом формулы (2.3).

Согласно формуле конечных прирашений Лагранжа при некотором $\theta,|\theta|<1$, имеем

$$
\begin{aligned}
{[\cdots] } & =(n-2 j)(r+1)\left(\frac{2 m+1}{2} n-\theta \frac{n-2 j}{2}\right)^{-(r+2)} \\
& <(n-2 j)(r+1)\left(\frac{2 m+1}{2} n-\frac{n-2 j}{2}\right)^{-(r+2)} \\
& <\frac{(n-2 j)(r+1)}{(m n)^{r+2}}<\frac{(n-2 j)(r+1)}{m^{r+2} n(2 \nu)^{r+1}}<\frac{n-2 j}{m^{r+2} n j^{r+1}} .
\end{aligned}
$$

Поэтому

$$
\sum_{m=1}^{\infty}|[\cdots]|<\sum_{m=1}^{\infty} \frac{n-2 j}{m^{r+2} n j^{r+1}}=O\left(\frac{n-2 j}{n j^{r+1}}\right)
$$

Лемма доказана.

Заметим, что из (2.3) следует, что для $j=1, \ldots, \nu$ справедлива оценка

$$
B_{r, j, n}=O\left(\frac{1}{j^{r+1}}\right) .
$$

ЛЕмма 3. При $р \geqslant 0$ справедливы равномернье относительно параметров ри о оченки

$$
\begin{array}{r}
\sum_{j=1}^{\nu} j^{p}=\frac{1}{p+1}(\nu+1)^{p+1}+O\left((\nu+1)^{p}\right), \\
\sum_{j=1}^{\nu}\left(1+(-1)^{j}\right) j^{p}=\frac{1}{p+1}(\nu+1)^{p+1}+O\left((\nu+1)^{p}\right) .
\end{array}
$$

ДоказАТЕЛьСтво. Сначала докажем оценку (2.5). Имеем

$$
\sum_{j=1}^{\nu} j^{p}<\int_{0}^{\nu+1} u^{p} d u=\frac{1}{p+1}(\nu+1)^{p+1}
$$

С другой стороны,

$$
\sum_{j=1}^{\nu} j^{p}=\sum_{j=1}^{\nu+1} j^{p}-(\nu+1)^{p}>\int_{0}^{\nu+1} u^{p} d u-(\nu+1)^{p}=\frac{1}{p+1}(\nu+1)^{p+1}-(\nu+1)^{p} .
$$


Эти две оценки приводят к (2.5).

При доказательстве (2.6) будем отдельно рассматривать случаи нечетных и четных $\nu$.

Если $\nu$ нечетно, $\nu=2 k+1$, то

$$
\sum_{j=1}^{\nu}\left(1+(-1)^{j}\right) j^{p}=\sum_{j=1}^{2 k}\left(1+(-1)^{j}\right) j^{p}=\sum_{i=1}^{k} 2 \cdot(2 i)^{p}=2^{p+1} \sum_{i=1}^{k} i^{p}
$$

Отсюда, пользуясь оценкой (2.5), находим

$$
\begin{aligned}
\sum_{j=1}^{\nu}\left(1+(-1)^{j}\right) j^{p} & =2^{p+1}\left(\frac{1}{p+1}(k+1)^{p+1}+O\left((k+1)^{p}\right)\right) \\
& =\frac{1}{p+1}(\nu+1)^{p+1}+O\left((\nu+1)^{p}\right)
\end{aligned}
$$

и мы получили (2.6).

Пусть теперь $\nu$ четно, $\nu=2 k$. Тогда

$$
\sum_{j=1}^{\nu}\left(1+(-1)^{j}\right) j^{p}=\sum_{j=1}^{2(k-1)}\left(1+(-1)^{j}\right) j^{p}+2 \nu^{p}=2^{p+1} \sum_{i=1}^{k-1} i^{p}+2 \nu^{p}
$$

и, вновь пользуясь (2.5), видим, что

$$
\sum_{j=1}^{\nu}\left(1+(-1)^{j}\right) j^{p}=2^{p+1}\left(\frac{1}{p+1} k^{p+1}+O\left(k^{p}\right)\right)+2 \nu^{p}=\frac{1}{p+1} \nu^{p+1}+O\left(\nu^{p}\right) .
$$

Чтобы прийти к (2.6), воспользуемся тем, что в силу формулы конечных прирашений Лагранжа

$$
(\nu+1)^{p+1}-\nu^{p+1}=(p+1)(\nu+\theta)^{p}<(p+1)(\nu+1)^{p}
$$

Лемма доказана.

ЛЕмма 4. Справедливъц равномерные относительно всех параметров оценки

$$
\begin{gathered}
\sum_{j=1}^{\nu} \frac{1}{\nu-j+1}\left[1-\left(\frac{j}{n-j}\right)^{r}\right]=\log \min (r, n)+O(1), \\
\sum_{j=1}^{\nu} \frac{1+(-1)^{j}}{\nu-j+1}\left[1-\left(\frac{j}{n-j}\right)^{r}\right]=\log \min (r, n)+O(1), \\
\sum_{j=1}^{\nu} \frac{1}{\nu-j+1}\left[1-\left(\frac{j}{n-j}\right)^{r}\right]\left(\frac{j}{\nu+1}\right)^{r}=O(1) .
\end{gathered}
$$


ДокаЗАтЕльство. Сначала оценим ошибку от замены $n-j$ в знаменателе дроби в $(2.7)$ на $\nu+1$. Пользуясь формулой конечных приращений Лагранжа, находим, что при некотором $\theta, 0<\theta<1$,

$$
\begin{aligned}
\frac{1}{(\nu+1)^{r}}-\frac{1}{(n-j)^{r}} & =\frac{(n-j)^{r}-(\nu+1)^{r}}{(\nu+1)^{r}(n-j)^{r}} \\
& =\frac{r(n-j-(\nu+1))(n-j-\theta(n-j-(\nu+1)))^{r-1}}{(\nu+1)^{r}(n-j)^{r}} \\
& <\frac{r(n-j-(\nu+1))}{(\nu+1)^{r+1}} .
\end{aligned}
$$

Но согласно $(1.1) n-j-(\nu+1) \leqslant \nu-j+1$. Поэтому ошибка от рассматриваемой замены в сумме из левой части формулы (2.7) не превосходит величины

$$
\sum_{j=1}^{\nu} \frac{1}{\nu-j+1} j^{r} \frac{r(n-j-(\nu+1))}{(\nu+1)^{r+1}} \leqslant \sum_{j=1}^{\nu} \frac{r j^{r}}{(\nu+1)^{r+1}}=O(1)
$$

Таким образом,

$$
\sum_{j=1}^{\nu} \frac{1}{\nu-j+1}\left[1-\left(\frac{j}{n-j}\right)^{r}\right]=\sum_{j=1}^{\nu} \frac{1}{\nu-j+1}\left[1-\left(\frac{j}{\nu+1}\right)^{r}\right]+O(1) .
$$

Проведенные рассуждения доказывают также, что

$$
\sum_{j=1}^{\nu} \frac{1+(-1)^{j}}{\nu-j+1}\left[1-\left(\frac{j}{n-j}\right)^{r}\right]=\sum_{j=1}^{\nu} \frac{1+(-1)^{j}}{\nu-j+1}\left[1-\left(\frac{j}{\nu+1}\right)^{r}\right]+O(1)
$$

и

$$
\begin{aligned}
\sum_{j=1}^{\nu} \frac{1}{\nu-j+1}[1- & \left.\left(\frac{j}{n-j}\right)^{r}\right]\left(\frac{j}{\nu+1}\right)^{r} \\
& =\sum_{j=1}^{\nu} \frac{1}{\nu-j+1}\left[1-\left(\frac{j}{\nu+1}\right)^{r}\right]\left(\frac{j}{\nu+1}\right)^{r}+O(1)
\end{aligned}
$$

Преобразуем сумму из правой части оценки (2.10). Имеем

$$
1-\left(\frac{j}{\nu+1}\right)^{r}=\left(1-\frac{j}{\nu+1}\right) \sum_{p=0}^{r-1}\left(\frac{j}{\nu+1}\right)^{p}=(\nu-j+1) \sum_{p=0}^{r-1} \frac{j^{p}}{(\nu+1)^{p+1}} .
$$

Поэтому

$$
\begin{aligned}
\sum_{j=1}^{\nu} \frac{1}{\nu-j+1}\left[1-\left(\frac{j}{\nu+1}\right)^{r}\right] & =\sum_{j=1}^{\nu} \sum_{p=0}^{r-1} \frac{j^{p}}{(\nu+1)^{p+1}} \\
& =\sum_{p=0}^{r-1} \frac{1}{(\nu+1)^{p+1}} \sum_{j=1}^{\nu} j^{p}
\end{aligned}
$$


Но в силу (2.5)

$\sum_{p=0}^{r-1} \frac{1}{(\nu+1)^{p+1}} \sum_{j=1}^{\nu} j^{p}=\sum_{p=0}^{r-1} \frac{1}{p+1}+O\left(\sum_{p=0}^{r-1} \frac{1}{\nu+1}\right)=\log r+O(1)+O\left(\frac{r}{\nu}\right)$

Для $r \leqslant \nu$ оценка (2.7) следует из (2.10), (2.13) и (2.14).

Для $r>\nu$, пользуясь этими же соотношениями, получаем

$$
\begin{aligned}
\sum_{j=1}^{\nu} & \frac{1}{\nu-j+1}\left[1-\left(\frac{j}{n-j}\right)^{r}\right] \\
& =\sum_{p=0}^{\nu-1} \frac{1}{(\nu+1)^{p+1}} \sum_{j=1}^{\nu} j^{p}+O(1)+O\left(\sum_{p=\nu}^{\infty} \frac{1}{(\nu+1)^{p+1}} \sum_{j=1}^{\nu} j^{p}\right) \\
& =\log \nu+O(1)+O\left(\sum_{p=\nu}^{\infty} \frac{1}{(\nu+1)^{p+1}} \sum_{j=1}^{\nu} j^{p}\right) .
\end{aligned}
$$

Покажем, что

$$
\sum_{p=\nu}^{\infty} \frac{1}{(\nu+1)^{p+1}} \sum_{j=1}^{\nu} j^{p}=O(1)
$$

Тогда оценка (2.7) для $r>\nu$ будет вытекать из (2.15).

Имеем

$$
\sum_{j=1}^{\nu} j^{p}<\nu^{p}+\int_{0}^{\nu} u^{p} d u=\nu^{p}+\frac{\nu^{p+1}}{p+1}
$$

Значит, для $p \geqslant \nu$

$$
\sum_{j=1}^{\nu} j^{p}<2 \nu^{p}
$$

Поэтому

$$
\sum_{p=\nu}^{\infty} \frac{1}{(\nu+1)^{p+1}} \sum_{j=1}^{\nu} j^{p}<\frac{2}{\nu+1} \sum_{p=\nu}^{\infty}\left(\frac{\nu}{\nu+1}\right)^{p}<\frac{2}{\nu+1}\left(1-\frac{\nu}{\nu+1}\right)^{-1}=2
$$

и мы получили (2.16).

Таким образом, оценка (2.7) доказана.

Для суммы из правой части оценки (2.11) аналог тождества (2.13) имеет вид

$$
\sum_{j=1}^{\nu} \frac{1+(-1)^{j}}{\nu-j+1}\left[1-\left(\frac{j}{\nu+1}\right)^{r}\right]=\sum_{p=0}^{r-1} \frac{1}{(\nu+1)^{p+1}} \sum_{j=1}^{\nu}\left(1+(-1)^{j}\right) j^{p}
$$

и пользуясь вместо (2.5) оценкой (2.6), с помощью тех же рассуждений приходим $\mathrm{K}(2.8)$. 
Наконец, для получения оценки (2.9) записываем аналог представления (2.13):

$$
\sum_{j=1}^{\nu} \frac{1}{\nu-j+1}\left[1-\left(\frac{j}{\nu+1}\right)^{r}\right]\left(\frac{j}{\nu+1}\right)^{r}=\sum_{p=0}^{r-1} \frac{1}{(\nu+1)^{p+r+1}} \sum_{j=1}^{\nu} j^{p+r},
$$

откуда согласно (2.5) вытекает, что

$$
\begin{aligned}
\sum_{j=1}^{\nu} \frac{1}{\nu-j+1}\left[1-\left(\frac{j}{\nu+1}\right)^{r}\right] & \left(\frac{j}{\nu+1}\right)^{r} \\
= & \sum_{p=0}^{r-1} \frac{1}{p+r+1}+O\left(\sum_{p=0}^{r-1} \frac{1}{\nu+1}\right)=O\left(1+\frac{r}{\nu}\right) .
\end{aligned}
$$

В силу (2.12) это доказьвает оценку (2.9) для $r \leqslant \nu$. А для $r>\nu$ с помощью $(2.5)$ и (2.16) получаем

$$
\begin{aligned}
& \sum_{p=0}^{r-1} \frac{1}{(\nu+1)^{p+r+1}} \sum_{j=1}^{\nu} j^{p+r} \\
& \quad<\sum_{p=0}^{\nu-1} \frac{1}{(\nu+1)^{p+r+1}} \sum_{j=1}^{\nu} j^{p+r}+\sum_{p=\nu}^{\infty} \frac{1}{(\nu+1)^{p+r+1}} \sum_{j=1}^{\nu} j^{p+r} \\
& \quad=\sum_{p=0}^{\nu-1} \frac{1}{p+r+1}+O(1)=O(1),
\end{aligned}
$$

что приводит к оценке (2.9).

Лемма доказана.

Заметим, что из (2.7) и (2.8) следует оценка

$$
\sum_{j=1}^{\nu} \frac{1-(-1)^{j}}{\nu-j+1}\left[1-\left(\frac{j}{n-j}\right)^{r}\right]=\log \min (r, n)+O(1) .
$$

\section{§3. Доказательство теоремы}

В качестве первого шага доказательства воспользуемся оценкой

$$
\frac{1}{\cos \frac{j \pi}{n}}=\frac{1}{\sin \left(\frac{\pi}{2 n}(n-2 j)\right)}=\frac{2 n}{\pi(n-2 j)}+O(1) .
$$

Подставив эту оценку в (1.3), получим

$$
L_{r, n}=\frac{2}{\pi} \sum_{j=1}^{\nu} \frac{2+(-1)^{j+\nu}+(-1)^{j+\nu+n}}{n-2 j} \frac{B_{r, j, n}}{A_{r, j, n}}+O\left(1+\frac{1}{n} \sum_{j=1}^{\nu} \frac{B_{r, j, n}}{A_{r, j, n}}\right) .
$$


В силу левой оценки (2.1) и оценки (2.4)

$$
\frac{1}{n} \sum_{j=1}^{\nu} \frac{B_{r, j, n}}{A_{r, j, n}}=O(1)
$$

Таким образом,

$$
L_{r, n}=\frac{2}{\pi} \sum_{j=1}^{\nu} \frac{2+(-1)^{j+\nu}+(-1)^{j+\nu+n}}{n-2 j} \frac{B_{r, j, n}}{A_{r, j, n}}+O(1)
$$

Заменим в (3.1) $B_{r, j, n}$ на

$$
\frac{1}{j^{r+1}}-\frac{1}{(n-j)^{r+1}}
$$

В силу (2.3) и левой оценки (2.1) ошибка от такой замены не превосходит (с точностью до абсолютной постоянной в качестве множителя) суммы

$$
\sum_{j=1}^{\nu} \frac{1}{(n-2 j) A_{r, j, n}} \cdot \frac{n-2 j}{n j^{r+1}}=\sum_{j=1}^{\nu} \frac{1}{A_{r, j, n} n j^{r+1}}<1
$$

Поэтому

$$
L_{r, n}=\frac{2}{\pi} \sum_{j=1}^{\nu} \frac{2+(-1)^{j+\nu}+(-1)^{j+\nu+n}}{(n-2 j) A_{r, j, n}}\left[\frac{1}{j^{r+1}}-\frac{1}{(n-j)^{r+1}}\right]+O(1)
$$

Так как в силу левой оценки (2.1)

$$
\frac{1}{A_{r, j, n}}\left[\frac{1}{j^{r+1}}-\frac{1}{(n-j)^{r+1}}\right]<1
$$

то, заменив в знаменателе дроби из (3.2) $n-2 j$ на $2(\nu-j+1)$, получим

$$
L_{r, n}=\frac{1}{\pi} \sum_{j=1}^{\nu} \frac{2+(-1)^{j+\nu}+(-1)^{j+\nu+n}}{(\nu-j+1) A_{r, j, n}}\left[\frac{1}{j^{r+1}}-\frac{1}{(n-j)^{r+1}}\right]+O(1)
$$

Из неравенств (2.1) следует, что при некотором $\theta, 0<\theta<1$,

$$
A_{r, j, n}=\frac{1}{j^{r+1}}+\frac{4 \theta}{(n-j)^{r+1}}
$$

Отсюда находим

$$
\frac{1}{A_{r, j, n}}=j^{r+1}+O\left(\frac{j^{2(r+1)}}{(n-j)^{r+1}}\right)
$$


Подставим эту оценку в (3.3). Тогда получим

$$
\begin{aligned}
& L_{r, n}=\frac{1}{\pi} \sum_{j=1}^{\nu} \frac{2+(-1)^{j+\nu}+(-1)^{j+\nu+n}}{\nu-j+1}\left[1-\left(\frac{j}{n-j}\right)^{r+1}\right] \\
&+O\left(\sum_{j=1}^{\nu} \frac{1}{\nu-j+1}\left[\left(\frac{j}{n-j}\right)^{r+1}-\left(\frac{j}{n-j}\right)^{2(r+1)}\right]\right) .
\end{aligned}
$$

Чтобы оценить остаточный член формулы (3.4), заметим, что $n-j \geqslant \nu+1$. Поэтому

$$
\begin{aligned}
\sum_{j=1}^{\nu} \frac{1}{\nu-j+1}\left[\left(\frac{j}{n-j}\right)^{r+1}\right. & \left.-\left(\frac{j}{n-j}\right)^{2(r+1)}\right] \\
& \leqslant \sum_{i=1}^{\nu} \frac{1}{\nu-j+1}\left[1-\left(\frac{j}{n-j}\right)^{r+1}\right]\left(\frac{j}{\nu+1}\right)^{r+1}
\end{aligned}
$$

а последняя сумма согласно (2.9) ограничена абсолютной постоянной.

Таким образом,

$$
L_{r, n}=\frac{1}{\pi} \sum_{j=1}^{\nu} \frac{2+(-1)^{j+\nu}+(-1)^{j+\nu+n}}{\nu-j+1}\left[1-\left(\frac{j}{n-j}\right)^{r+1}\right]+O(1) .
$$

Если число $n$ нечетно, то $2+(-1)^{j+\nu}+(-1)^{j+\nu+n}=2$ и оценка (1.3) следует из (3.5) и (2.7).

Если же $n$ четно, то $2+(-1)^{j+\nu}+(-1)^{j+\nu+n}=2+2(-1)^{j+\nu}$. Таким образом, в этом случае оценка (1.3) вытекает из (3.5), (2.8) и (2.17).

Теорема доказана.

\section{Список литературы}

1. Субботин Ю. Н. О связи между конечньми разностями и соответствующими производными // Труды МИАН. 1965. Т. 78. С. 24-42.

2. Женсыкбаев $A . A$. Точные оценки равномерного приближения непрерьвных периодических функций сплайнами $r$-го порядка // Матем. заметки. 1973. Т. 13. № 2. С. 217-228.

3. Richards F. B. Best bounds for the uniform periodic spline interpolation operator // J. Approx. Theory. 1973. V. 7. P. 302-317.

4. Женсыкбаев А. А. Замечание о константах Лебега оператора сплайн-интерполяции // Исследования по современнњм проблемам суммирования и приближения функций и их приложениям. Днепропетровск: Днепропетр. гос. ун-т, 1974. Вьп. 5. С. 50-52.

5. Richards $F$. The Lebesque constants for cardinal spline interpolation // J. Approx. Theory. 1975. V. 14. P. 83-92.

6. Meinardus G. Über die Norm des Operators der Kardinalen Spline-Interpolation // J. Approx. Theory. 1976. V. 16. P. 289-298.

Институт математики и механики УрО РАН; 